\title{
Isotopic Analysis of NUSIMEP-6 Uranium Particles using SEM-TIMS
}

\author{
Jong-Ho Park*, Sujin Park, and Kyuseok Song \\ Nuclear Chemistry Research Division, Korea Atomic Energy Research Institute, Daejeon 305-353, Korea. \\ Received September 11, 2013; Revised September 24, 2013; Accepted September 24, 2013 \\ First published on the web September 30, 2013; DOI: 10.5478/MSL.2013.4.3.51
}

\begin{abstract}
Isotopic analysis using thermal ionization mass spectrometry coupled with scanning electron microscopy (SEMTIMS) was performed to determine the isotopic ratios of uranium contained in micro-particles in the 6th Nuclear Signatures Interlaboratory Measurement Evaluation Programme (NUSIMEP-6) sample. Elemental analysis by energy dispersive X-ray spectroscopy (EDS) was conducted on uranium-bearing mirco-particles, which were transferred to rhenium filaments for TIMS loading using a micromanipulation system in a SEM. A multi-ion-counter system was utilized to detect the ion signals of the four isotopes of uranium simultaneously. The isotope ratios of uranium corrected by bracketing using a reference material showed excellent agreement with the certified values. The measurement accuracy for $n\left({ }^{234} U\right) / n\left({ }^{238} U\right)$ and $(b) n\left({ }^{235} U\right) / n\left({ }^{238} U\right)$ was $10 \%$ and $1 \%$, respectively, which met the requirements for qualification for the NetWork of Analytical Laboratories (NWAL).
\end{abstract}

Keywords: Uranium, SEM-TIMS, Particle Analysis, Isotopic Analysis, Safeguards

\section{Introduction}

Highly accurate and precise isotopic analysis of nuclear materials in environmental samples plays an important role in monitoring the nuclear activity of a related facility for nuclear safeguards purpose. ${ }^{1,2}$ Authorized inspectors collect the nuclear material samples from the environment of a facility, which are called "swipe samples". The swipe samples may be subjected to two different types of analysis for nuclear safeguards depending on the analytical objectives: bulk analysis and particle analysis. The total amount and the overall isotopic ratios of the nuclear materials contained in a swipe sample are determined by bulk analysis. This process consists of the recovery of the nuclear materials from a swipe sample; the acid digestion of the recovered materials; the chemical separation of uranium, plutonium, and the other elements; and isotopic measurement generally using a mass spectrometer with high sensitivity. An isotope dilution mass spectrometry (IDMS) technique with a spiked reference material added to a portion of the sample is used for the quantification of uranium and/or plutonium. The typical amounts of nuclear materials in a swipe sample are approximately $10 \mathrm{ng}$ of uranium and/or $10 \mathrm{pg}$ of plutonium. The information from the bulk analysis provides a rough clue to the existence of undeclared nuclear activities.

In contrast to the bulk analysis, particle analysis enables the acquisition of the isotopic ratios of uranium and/or plutonium in the individual particles contained in a swipe

\footnotetext{
*Reprint requests to Dr. Jong-Ho Park
}

E-mail: jongho@kaeri.re.kr sample, which provides decisive information with which to track the nuclear history of a facility. Particle analysis requires a series of procedures consisting of particle recovery, target particle identification (or screening), particle handling, and isotopic measurements.

Particle analysis can be performed using the three different mass spectrometry techniques such as secondary ionization mass spectrometry (SIMS), thermal ionization mass spectrometry coupled with a fission track technique (FT-TIMS), and thermal ionization mass spectrometry coupled with a scanning electron microscopy technique (SEM-TIMS). The main criterion for the classification is how the target particles are found, which is a required process because most particles in a swipe sample are not particles bearing nuclear materials but rather non-nuclear dust consisting mainly of carbon.

In the SIMS technique, ${ }^{3,4}$ the particles in a swipe sample are collected in an electrically conductive carbon planchet by vacuum impaction followed by loading in the mass spectrometer. An automatic particle search function with low intensity of the primary ion beam irradiation determines the location of the target particles, and then, the isotopic measurement is performed directly on them. The SIMS technique is simple, but the screening and the measurement sensitivity are low compared to those of FTTIMS and SEM-TIMS.

The particles are recovered in a micro-porous filter by vacuum impaction in the FT-TIMS technique. Few additional processes are required to prepare a particle layer with an FT detector, to irradiate the thermal neutron from a nuclear reactor inducing the fission of nuclear materials, and to identify the location of target particles by searching the fission tracks on the detector. ${ }^{5,6}$ Delicate micromani- 
pulation is performed to transfer the particle- bearing layer to a measurement device; this is followed by isotopic measurements of the loaded particles utilizing a thermal ionization mass spectrometer (TIMS). The FT-TIMS technique is the most sensitive, accurate, and precise in terms of particle screening and isotopic measurement. However, the procedure is complicated relative to other techniques, and a nuclear reactor is required for the thermal neutron irradiation.

The particle recovery process used for the SIMS technique is also adopted for the SEM-TIMS technique. The planchet containing the collected particles is loaded into a secondary electron microscope (SEM) equipped with an energy dispersive X-ray spectrometer (EDS) and a micromanipulation system. Elemental analysis of the particles using EDS is performed to find the target particles, followed by the transfer of a particle into the TIMS for isotopic measurement. SEM-TIMS compensates for the disadvantages of SIMS and FT-TIMS by adopting a simple particle screening process and a sensitive, accurate, and precise isotopic measurement by TIMS. Therefore, it excludes the need for a nuclear reactor, and it relatively quickly obtains a final result with high accuracy and precision.

The Nuclear Signatures Interlaboratory Measurement Evaluation Programme (NUSIMEP) facilitates interlaboratory comparison among laboratories that perform uranium analyses. The 6th NUSIMEP (NUSIMEP-6) was opened in 2008 , focusing on the measurement of uranium isotope ratios in sub-micrometer uranium particles. Although the Korea Atomic Energy Research Institute (KAERI) did not participate, the sample was acquired once the program completed for self-test purposes.

In this paper, the isotopic analysis of the NUSIMEP-6 sample using the SEM-TIMS technique is described. A SEM equipped with an EDS was utilized to identify the uranium particles by elemental analysis. Micromanipulation of individual target particles was performed to load them into a TIMS for isotopic measurements. The multi-ion-counter system in the TIMS allowed us to detect four isotopes of uranium contained in the particles simultaneously and demonstrated excellent agreement between the measured isotope ratios and the certified values.

\section{Experimental}

The planchet containing the NUSIMEP-6 particles was introduced into a SEM (JSM-6610LV, JEOL) equipped with an EDS (X-Max, Oxford) for particle screening by elemental analysis. As shown in Figure 1, the microprobe of a threeaxis micromanipulator system (MM3M-EM, Kleindiek Nanotechnik) picked up an identified target particle from the planchet (a) and transferred it onto a zone-refined rhenium filament (Thermo Scientific) in which the uranium background was minimized $(b, c){ }^{7}$ The particle sample was

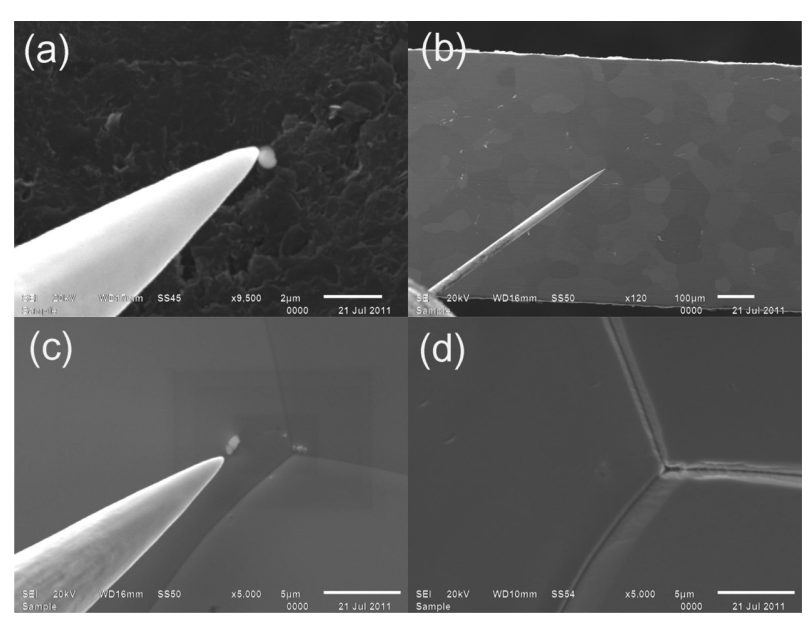

Figure 1. Micromanipulation of a uranium particle. Particle pick up (a), transfer (b) and loading (c) to a rhenium filament. SEM image after fixing (d).

fixed on the filament by adding $1 \mu \mathrm{L}$ of $1 \mathrm{M} \mathrm{HNO}_{3}$ and applying $1.8 \mathrm{~A}$ of current using a fixing device (d).

The experimental setup for the isotopic measurements using a TIMS (TRITON, Thermo Scientific), the detector configuration, and the adjustment of the detection efficiency of individual ion counters were described in detail elsewhere. ${ }^{7,89}$ Four channeltrons in a multiple ion counter (MIC) system were used to detect the ion signals of the four isotopes of uranium simultaneously.

The mass bias and background signal intensity were corrected using the bracketing technique. A bracketing correction factor $\left(\mathrm{C}_{\mathrm{b}}\right)$, which was determined by the isotopic measurement of a reference material (NIST U010), was applied to the isotopic measurements of the particle samples as follows:

$$
\begin{aligned}
& \mathrm{C}_{\mathrm{b}}=\mathrm{R}_{\text {cert_ref }} / \mathrm{R}_{\text {meas_ref }} \\
& \mathrm{R} \text { cor }=\mathrm{C}_{\mathrm{b}} \cdot \mathrm{R}_{\text {meas }}
\end{aligned}
$$

where $R_{\text {cert_ref }}$ and $R_{\text {meas ref }}$ are the certified and the measured isotope ratios of the reference material, respectively, and $\mathrm{R}_{\text {cor }}$ and $\mathrm{R}_{\text {meas }}$ are the corrected and the measured isotope ratios of the samples, respectively. For bracketing, the ion signal intensity of U-238 in the measurement of the reference material was regulated to approximately $10,000 \mathrm{cps}$, which was comparable to the actual measurement of a NUSIMEP-6 particle.

A double-filament system, which decouples the evaporation and the ionization of the sample, was adopted with the continuous heating method. ${ }^{10}$ The weights for the individual data sets were considered to calculate the weighted mean and the weighted standard error of the isotopic measurements. The uncertainty was estimated according to GUM (Guide to the expression of Uncertainty in Measurement). ${ }^{11}$ 


\section{Results and Discussion}

Figure 2 (a) shows the SEM image of a porting of the NUSIMEP-6 sample. A considerable number of microparticles, mostly sub-micron in diameter, were observed in the image. However, only the particle circled in the figure were identified as the particles bearing uranium by the uranium mapping(Figure 2 (b)). Elemental analysis by the localized EDS was performed on individual particles resulting in the intense $\mathrm{M}-\alpha$ line at approximately 3.2 and $3.3 \mathrm{keV}$ from the particles in the circles (Figure 3), but no such spectrum was obtained from the other particles.

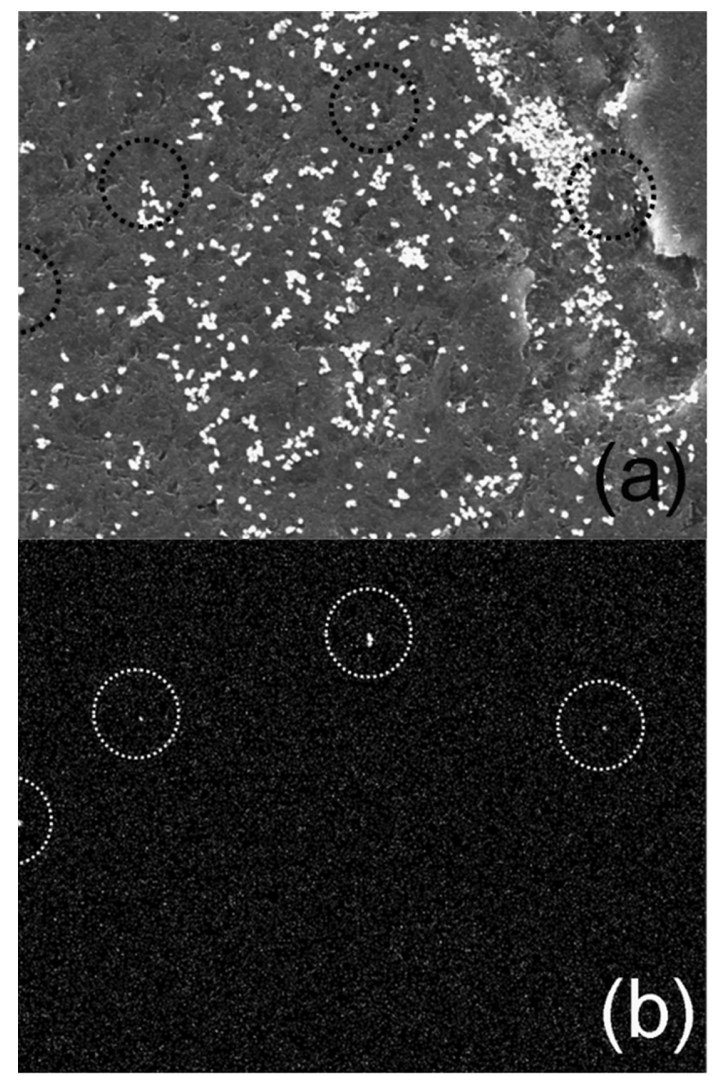

Figure 2. SEM image of a portion of the NUSIMEP-6 sample (a) and the uranium mapping image by EDS (b).

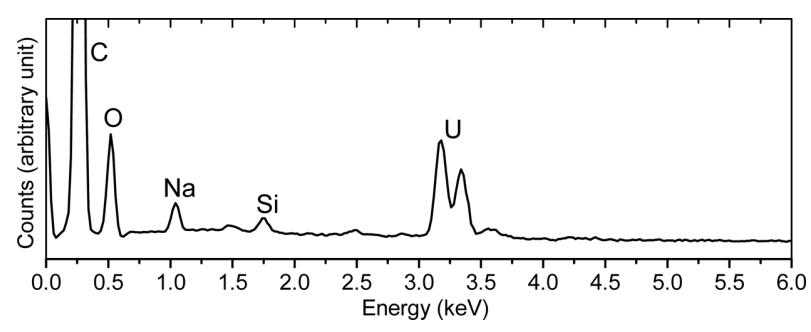

Figure 3. The EDS spectrum of an identified uranium particle in the NUSIMEP-6.
However, this does not mean that the other particles were excluded from the nuclear particles because the elemental analysis by EDS is not sufficiently sensitive for microparticles with sub-micron sizes. Additional nuclear particles could have been identified using the FT technique, but this would have required further processing, including thermal neutron irradiation, and at least several additional days.

Assuming that the chemical forms of uranium in the particles are uranium oxides, such as $\mathrm{UO}_{2}$ or $\mathrm{U}_{3} \mathrm{O}_{8}$, the amount of uranium in a particle of $0.6 \mu \mathrm{m}$ average size, is estimated to be approximately $1 \mathrm{pg}$. For the measurement of the sample in ultra-trace amounts, the accuracy and the precision of the TIMS measurements were greatly enhanced when the simultaneous measurement method was used rather than the multi-dynamic measurement, as discussed previously. ${ }^{9}$ Therefore, instead of Faraday cups and a secondary electron multiplier, a MIC system of the TIMS was utilized for the isotopic measurement of the target particles with sub-micron sizes.

Table 1 shows the isotopic ratios $\left(n\left({ }^{234} \mathrm{U}\right) / \mathrm{n}\left({ }^{238} \mathrm{U}\right)\right.$ and $\left.\mathrm{n}\left({ }^{235} \mathrm{U}\right) / \mathrm{n}\left({ }^{238} \mathrm{U}\right)\right)$ of uranium in the screened uranium particles of the NUSIMEP-6 sample. The values of $n\left({ }^{236} \mathrm{U}\right) /$ $\mathrm{n}\left({ }^{238} \mathrm{U}\right)$ were not listed due to the extremely low abundance of $\mathrm{U}-236$, which was on the order of $10^{-7}$. The certified values of each ratio are also shown in the table.

The accuracy, defined as the deviation from the certified values, was within $10 \%$ and $1 \%$ for $n\left({ }^{234} \mathrm{U}\right) / \mathrm{n}\left({ }^{238} \mathrm{U}\right)$ and $n\left({ }^{235} U\right) / n\left({ }^{238} U\right)$, respectively. Considering the guidelines of the International Atomic Energy Agency (IAEA), the accuracy met the requirement for qualification for the NetWork of Analytical Laboratories (NWAL) for analysis of particle environmental samples using FT-TIMS. It is noted that the amounts of U-234 and U-235 in a particle are estimated to be $40 \mathrm{ag}$ and $7 \mathrm{fg}$, respectively.

The analytical results are plotted in Figure 4, along with the certified values (in dashed line). The measured isotopic ratios were in agreement with the certified values within the uncertainties.

Although the channeltrons in the MIC system offer great performance for the simultaneous measurement of the nuclear materials at ultra-trace levels, a considerable lack of

Table 1. Uranium isotope ratios of the NUSIMP-6 microparticles

\begin{tabular}{ccc}
\hline \hline & $\mathrm{n}\left({ }^{234} \mathrm{U}\right) / \mathrm{n}\left({ }^{238} \mathrm{U}\right)$ & $\mathrm{n}\left({ }^{235} \mathrm{U}\right) / \mathrm{n}\left({ }^{238} \mathrm{U}\right)$ \\
\hline Certified Value & $0.000049817(48)$ & $0.0070439(35)$ \\
Particle \#1 & $0.000048(10)$ & $0.00703(10)$ \\
Particle \#2 & $0.000047(10)$ & $0.00709(14)$ \\
Particle \#3 & $0.000044(14)$ & $0.00711(21)$ \\
Particle \#4 & $0.000045(14)$ & $0.00705(10)$ \\
Particle \#5 & $0.000051(22)$ & $0.00704(17)$ \\
Particle \#6 & $0.000044(11)$ & $0.00699(16)$ \\
\hline
\end{tabular}

Numbers in parentheses indicate expanded uncertainties $U=k \cdot u_{c}$ 

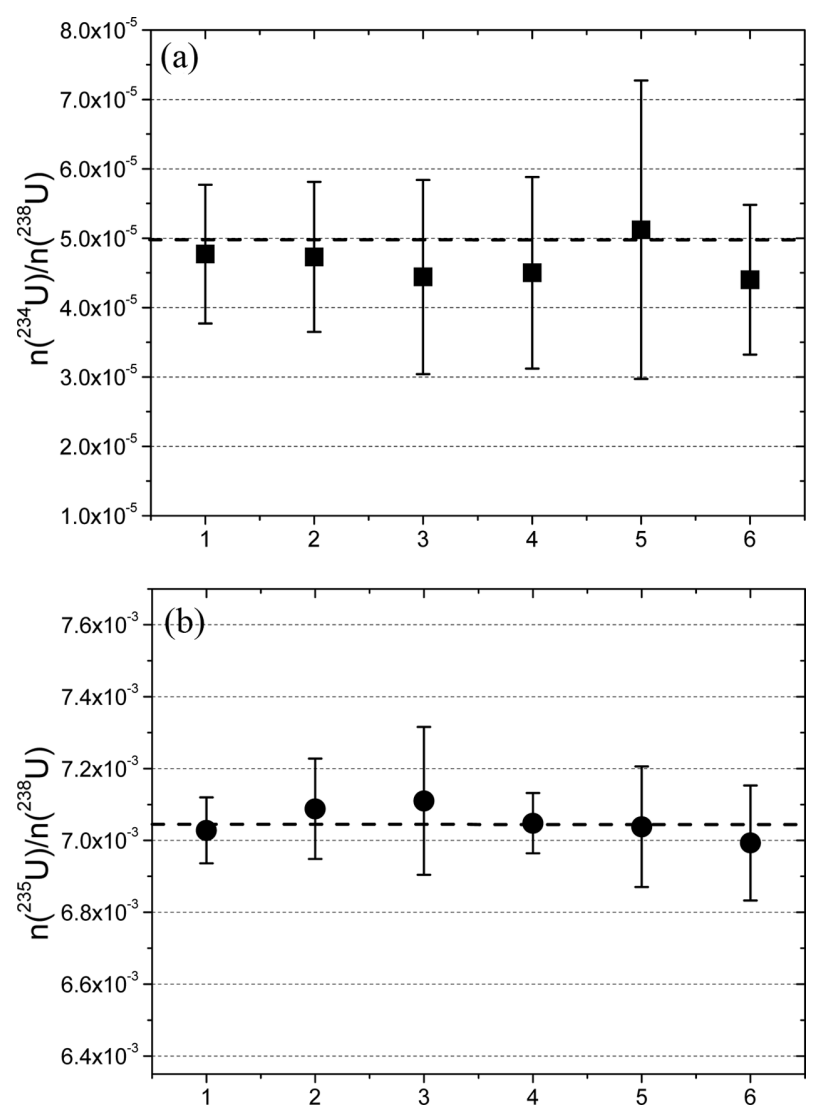

Figure 4. Uranium isotope ratios of NUSIMEP-6 microparticles; (a) $n\left({ }^{234} U\right) / n\left({ }^{238} U\right)$, and (b) $n\left({ }^{235} U\right) / n\left({ }^{238} U\right)$.

stability was observed. Because the detection efficiency of the channeltrons decreased relatively quickly, the reference measurements for bracketing needed to be performed prior to every measurement. Recently, a new TIMS with different types of ion counters, which offer greater stability than the channeltrons, was installed in KAERI. Further studies and measurements of micro-particles will be conducted using the new multi ion counter system, which is expected to have higher accuracy and precision than the current study.

\section{Summary}

The SEM-TIMS technique was used for the isotopic analysis of uranium-bearing micro-particles contained in a
NUSIMEP-6 sample. To identify target particles, elemental analysis using a SEM-EDS was performed on the planchet sample. Delicate micromanipulation of the identified particles enabled us to load them on TIMS filaments. Because the individual particle contained an ultra-trace amount of uranium, approximately $1 \mathrm{pg}$, the simultaneous measurement method was adopted for the isotopic measurement to enhance the accuracy and the precision. The measurement accuracy was $10 \%$ and $1 \%$ for $n\left({ }^{234} \mathrm{U}\right) /$ $n\left({ }^{238} U\right)$ and (b) $n\left({ }^{235} U\right) / n\left({ }^{238} U\right)$, respectively, which showed excellent agreement with the certified values.

\section{Acknowledgements}

The authors acknowledge the financial support for this study from the Nuclear Safety and Security Commission of the Korean government.

\section{References}

1. Donohue, D. L. J. Alloy Compd. 1998, 271-273, 11.

2. Heumann, K. G.; Eisenhut, S.; Gallus, S.; Hebeda, E. H.; Nusko, R.; Vengosh, A.; Walczyk, T. Analyst 1995, 120, 1291.

3. Esaka, F.; Magara, M.; Lee, C. G.; Sakurai, S.; Usuda, S.; Shinohara, N. Talanta 2009, 78, 290.

4. Esaka, F.; Magara, M.; Suzuki, D.; Miyamoto, Y.; Lee, C. G.; Kimura, T. Mass Spectrom. Lett. 2011, 2, 80.

5. Lee, C. G.; Iguchi, K.; Esaka, F.; Magara, M.; Sakurai, S.; Watanabe, K.; Usuda, S. Jpn. J. Appl. Phys. 2006, 45, 294.

6. Esaka, K. T.; Esaka, F.; Inagawa, J.; Iguchi, K.; Lee, C. G.; Sakurai, S.; Watanabe, K.; Usuda, S. Jpn, J. Appl. Phys. 2004, 43, L915-L9164.

7. Park, J.-H.; Choi, I.; Song, K. Mass Spectrom. Lett. 2010, $1,17$.

8. Park, J.-H.; Choi, I.; Park, S.; Lee, M.; Song, K. Bull. Korean Chem. Soc. 2012, 32, 4327.

9. Park, J.-H.; Jeong, K.; Song, K. Asian J. Chem. 2013, 25, 7061.

10. Suzuki, D.; Kokubu, Y. S.; Sakurai, S.; Lee, C. G.; Magara, M.; Iguchi, K.; Kimura, T. Int. J. Mass. Spectrom. 2010, 294, 23.

11. Bürger, S.; Essex, R. M.; Mathew, K. J.; Richter, S.; Thomas, R. B. Int. J. Mass Spectrom. 2010, 294, 65. 